\title{
Design and Technical Evaluation of an Enhanced Location-Awareness Service Enabler for Spatial Disorientation Management of Elderly With Mild Cognitive Impairment
}

\author{
Pedro A. Moreno, M. Elena Hernando
}

and Enrique J. Gómez

\begin{abstract}
The progressive ageing of population has turned the mild cognitive impairment (MCI) into a prevalent disease suffered by elderly. Consequently, the spatial disorientation has become a significant problem for older people and their caregivers. The ambient-assisted living applications are offering location-based services for empowering elderly to go outside and encouraging a greater independence. Therefore, this paper describes the design and technical evaluation of a location-awareness service enabler aimed at supporting and managing probable wandering situations of a person with MCI. Through the presence capabilities of the IP multimedia subsystem (IMS) architecture, the service will alert patient's contacts if a hazardous situation is detected depending on his location. Furthermore, information about the older person's security areas has been included in the user profile managed by IMS. In doing so, the service enabler introduced contribute to "contextawareness" paradigm allowing the adaptation and personalization of services depending on user's context and specific conditions or preferences.
\end{abstract}

Index Terms-Context-aware, dementia wandering support, IMS presence service, personalized location-based services.

\section{INTRODUCTION}

$\mathbf{N}$ OWADAYS elderly population is increasing in developed countries and new sociohealthcare services are being demanded to maintain the older adult's quality of life and autonomy in their own home and community. Home automation, telehealth services, and ambient intelligence are increasingly becoming elderly centered tools to support older adults. In particular, the European program ambient-assisted living [1] promotes the creation of products, systems, and solutions based on Information and Communication Technologies for elderly's life independence support.

Older age is associated with functional decline in selective aspects of cognitive performance (executive functions, attention verbal, visual memory, spatial navigation, and processing speed). A survey exposes that up to $60 \%$ of people with mild cognitive impairment (MCI) will experience any spatial disorientation incident ("getting lost" or "wandering") and it increases the involvement in hazardous situations [2]. Being aware of wandering risks of a person with MCI (PwMCI), the caregivers suffer an increased distress. Also, they are involved in the conflict between the prevention of wandering and the PwMCI's right to independence. This situation precipitates the institutionalization of the PwMCI.

Currently, devices employed to connect to the Internet are becoming more mobile, and they allow extracting user's information or specific preference/conditions to provide personalized services as the "context-awareness" paradigm establishes. Particularly, user's location information becomes a critical piece of context to customize "location-awareness" applications. The location-based services (LBS) imply an added value to thirdparty applications and are poised to become the next innovation in mobile phone. Therefore, the employment of new tracking and communication technologies in LBS will allow PwMCI, who have been stigmatized through the diagnosis of MCI, to go outside with more independence and reduce their institutionalization time. By monitoring the patient's location, their caregivers will be able to manage wandering situations, and reduce probable anxiety and psychological burden [4]. Thus, these solutions will allow substituting current practices (e.g., strong medication or mobility restriction) that deteriorate the autonomy of a PwMCI, and decrease their self-esteem and quality of life.

The main structure of a LBS is based upon a number of core components as: location-based applications running on mobile devices; handset location measurement technology (e.g., Cell ID, GPS); location management platforms; and server side daemons (navigation systems, tracking systems or emergency systems). The reliability of location technologies in LBS is a key issue that should be considered since the quality of service depends on the accuracy of mobile user's location information. For example, GPS systems can accurately locate an object but Cell ID mechanism is a better alternative to indoor location. In particular, Cell ID employs the geographical coverage of mobile network's base stations to calculate the device's location [5]. In addition, mature standards, architectures, and servers resources in LBS are needed to support an increasing number of subscribers, and to achieve a high integration between the different stakeholders.

This study presents an enhanced location-awareness service aimed at detecting wandering episodes of a PwMCI in specific context situations. If disorientation occurs, the service will 
activate dynamic alerts and notify to older adult's contacts. The service has been developed using the presence functionality of IP multimedia subsystem (IMS) architecture due to its capabilities of interoperability, standard compliant, service modularity, and horizontal architecture.

This paper contains five sections. Section I consists of an introduction. In Section II, previous works related to LBS focused on wandering are described. The technologies and components used in the service development as well as the service's functionalities are described in Section III. Section IV describes the results of the experiments carried out. Finally, Section V presents discussions and conclusions.

\section{RELATED WORKS}

In locations-based services (LBS) domain, one of the most common services is search engines that consider the subscriber's location and other issues as local time, weather, traffic, maps, and directions [6]. As examples of the "location-awareness" paradigm in e-health field, certain applications employ LBS to send health information or reminders depending on the user's location; monitor patients ubiquitously for managing medical emergencies; show environmental values dangerous to user's health; locate the nearest hospital facilities or staff; or trace the routes taken by people infected [7].

Particularly, some experiences use LBS to track people with MCI. The system described in [8] uses a GPS-enabled mobile phone to locate missing persons with a high reliability and accuracy due to the stability of the central server that handles the location service. The OutCare [9] and iRoute [10] systems monitor subconsciously if the patient deviates from a reasoned predicted route; try to guide the person back on track; and inform to his relatives. A similar approach is implemented in $\mathrm{LaCaSa}$ system that applies Markov models on the PwMCI context information (current location, noise or known locations) to infer if the patient is wandering [11]. In addition, the iWander project [12] presents an application capable of determining if the person is wandering depending on device's GPS sensor, time of day, weather condition, user feedback, and safe zones. It takes actions that navigate the patient to a safe location, notify caregivers, provide the current location, and call 911. The OAED system introduces another procedure based on GPS traces of the PwMCI to estimate if he/she is wandering [13]. In [14], the KITE project facilitates the communication between person with MCI and their families in case of wandering. Apart from GPS position technology, RFID [15] or WIFI [16] is used to avoid uncontrolled movement in their daily life indoor areas, getting lost or accessing dangerous areas.

Additionally, the study [17] reflects some requirements in the adoption of devices employed in LBS by PwMCI and his/her caregivers as: low cost, no need for training and support, technical and usability simplicity, size of equipment (a device as unnoticeable as possible), battery issues, location accuracy, presence of desired features, privacy issues, and avoid the stigma of being tagged. Apart from these previous considerations, LBS should provide features in order to allow the communication between caregivers and elderly, especially during the searching phase.
Regarding the use of IMS in LBS, a location emergency service is deployed in the architecture employing physical user data (pulse, body temperature, blood pressure) [18]. Furthermore, an IMS framework that allows sharing location between users or sending messages to users placed in a specific location is presented in [19].

In this study, the enhanced location-awareness service enabler developed uses IMS presence service to send the location information of a user (PwMCI) in a certain structured format with the aim at personalizing the service's functions. Moreover, the employment of session initiation protocol (SIP) in IMS enhances the enabler interoperability with other services. Finally, the service enabler provides a set of dynamic alerts considering other factors of patient's context than the previous described experiences (traveling, safety sense and day time, contacts companion).

\section{MATERIAL AND METHODS}

\section{A. IP Multimedia Subsystem}

Since networks operators do not want to be relegated to just an organizer of "bit pipes," they are promoting the IMS architecture as standard to offer interactive and real-time IP multimedia user services accessible from any place and any device. The horizontality of the IMS architecture is based on a group of common functions called "service enablers" that can be reused many times by several services independently on the device and access networks. These service enablers can implement application building blocks that represent basic "macro" capabilities common to many third-party services.

In order to allow multimedia session management, IMS employs the SIP for signaling functions. As physical structure, the entities responsible of signaling are a group of SIP servers, named Call Session Control Function (I-CSCF, P-CSCF and SCSCF), and a home subscriber server (HSS). These components manage user register and access; authorize the session's multimedia resources; and enable "triggers" to activate value-added services by means of initial filter criteria [20].

As location user data can be potentially confidential, users demand guarantee of privacy and security. Thus, IMS is suitable to deploy and offer the location enabler developed in this study because of the great confidence users have in their operators who are the main providers of the architecture.

\section{B. IMS Presence Service}

The IMS Presence Service is implemented in an added value application server, named presence server. The presence model defines presence as the information published by certain users, referred as presentities, toward other users, watchers, indicating their communication capabilities. The IMS architecture uses SIP/SIMPLE protocol for publishing, subscription, and notifying users' information through events definition. Therefore, the presentities publish changes in their information by using PUBLISH method, meanwhile watchers use SUBSCRIBE and NOTIFY methods to receive the updated presence information [20]. The presence information is described by means of a XML 


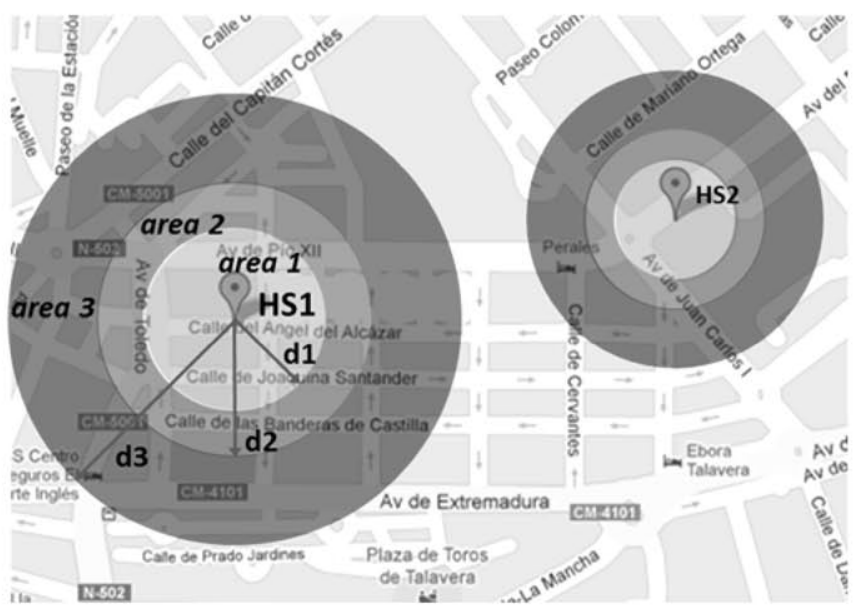

Fig. 1. Security-areas example (HS: Hot Spot; d: distance).

document with presence information data format (PIDF) format specified by SIP/SIMPLE [21]. Since PIDF format only allows giving information about the contact and its state, in this study we use the GEOPRIV [22] extension to include the user's geolocation to the presence information.

Moreover, the presence server can request or update user's profile data in a XML Document Management Server (XDMS) by using the XML Configuration Access Protocol (XCAP). The XDMS stores user's information complying with appusages, which refer to the XML data structure of user's profile. The server allows storing as much appusages as needed information (contact list, user's images).

\section{Location-Awareness Service Enabler Design}

The main contribution of this study is the design of a service enabler for an enhanced wandering management of PwMCI. The enabler uses the IMS presence service to monitor transparently the person's location; detect potential spatial disorientation or wandering events; which happen when the patient would not know the place where she/he is; and hence activates a set of alarms. The inference of these wandering episodes and the alerts are based on patient and contacts' context.

1) Security Areas Configuration: A new methodology named "Hot Spots (HSs)" has been defined to establish the security areas where a PwMCI could suffer a wandering episode. This method links particular geographical locations with places related to the patient (home, relative's house, nursing home, etc.). Taking these locations as references, a group of distances (d1, $\mathrm{d} 2$, and $\mathrm{d} 3$ ) are defined in order to delimit three security areas. The "area 1" defines the area where the patient usually develops his daily life activities. The second area refers to a caution area where the patient does not move regularly. This area could involve a wandering episode if the person moves further. Finally, the distance $\mathrm{d} 3$ determines the area where the PwMCI could suffer a spatial disorientation as the zone is totally unfamiliar to them. Fig. 1 shows the structure of these areas.
To include the information about the security areas in each patient's profile stored in the XDMS server, an appussage named "security areas" has been defined and its structure is as follows:

<ns: security-areas xmlns:ns='security-areas'

xsi:schemaLocation=' security-areas. $x$ sd' >

$<$ ns:area name="home">

<ns:list name="Contacts">

<ns:entry uri="sip: daugtheregbttalk.org">

$<$ ns:display-name>Helen</ns:display-name>

$</$ ns:entry $>$

<ns:entry uri="sip:sonegbttalk.org">

$<$ ns:display-name $>$ John $</$ ns:display-name $>$

$</$ ns:entry $></$ ns:list $>$

$<$ ns:circlesecurity $>$

$<$ ns:centerposition>

<ns: latitude $>37: 46: 30 \mathrm{~N}</ \mathrm{ns}$ : latitude $>$

$<$ ns:longitude $>122: 25: 20 \mathrm{~W}</ \mathrm{ns}:$ longitude $>$

$</$ ns: centerPosition $>$

$<$ ns: radius $>150</$ ns: radius $>$

$</$ ns:circlesecurity $>$

$<$ ns: radius Two $>200</$ ns : radius Two $>$

$<$ ns: radiusThree $>225</$ ns : radiusThree $>$

$<$ ns:timeSurveillance $>$

$<$ ns:timeslot name="sleep">

$<$ ns: $\min >20: 00: 00</ n s: \min >$

$<$ ns: $\max >09: 00: 00</$ ns: $\max >$

$</$ ns:timeSlot $></$ ns:timeSurveillance $>$

$</$ ns:area $></$ ns: security-areas $>$

The document can have multiple security areas that could be defined by a patient's contact (doctor or a closest relative) who know the places where patient usually moves. The items included for each security area (e.g., $<$ ns: area name $=$ "home" $>$ ) are: a list of contacts to notify the patient's situation ( $<$ ns:list $>$ ); the location data needed to establish the hot spot, i.e., HS in Fig. $1(<$ ns:centerPosition $>)$; the distances that conform the security areas $(<$ radius $>,<$ radiusTwo $>$ and $<$ radiusThree $>$ ); and a series of time slot that defines a period when the patient should not go out from a certain area. Note that the contacts would be involved in the document considering their will to be included in the contact list.

2) Enabler's Functionalities: The location enabler's functions and their relationship, depicted in Fig. 2, are described as follows.

a) Automatic Patient's Subscription: By a set of filter criteria, the IMS Core detects a patient's register and inform to the enabler which will subscribe to patient's presence information.

b) Patient's Information Processing: When receiving the patient's information in a NOTIFY (step 1), the enabler extracts from the XML certain information like current patient's location, speed, and the time that are based on GEOPRIV and PIDF as follows (steps 2 and 3 ):

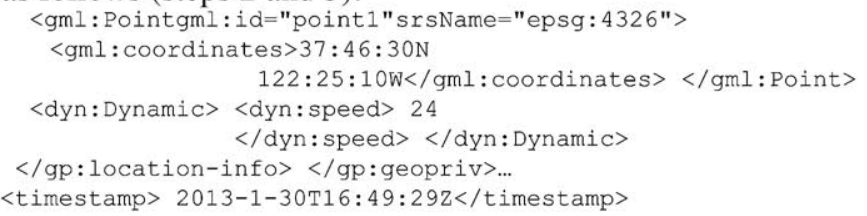

Next, the enabler asks to the XDMS server the "securityusage" appusage of the PwMCI. Thus, the list of contacts and the security-areas structure is extracted allowing the enabler to subscribe to contacts' presence information (step 4 and 6). The enabler will distinguish if the NOTIFY is coming from the patient or a contact. 


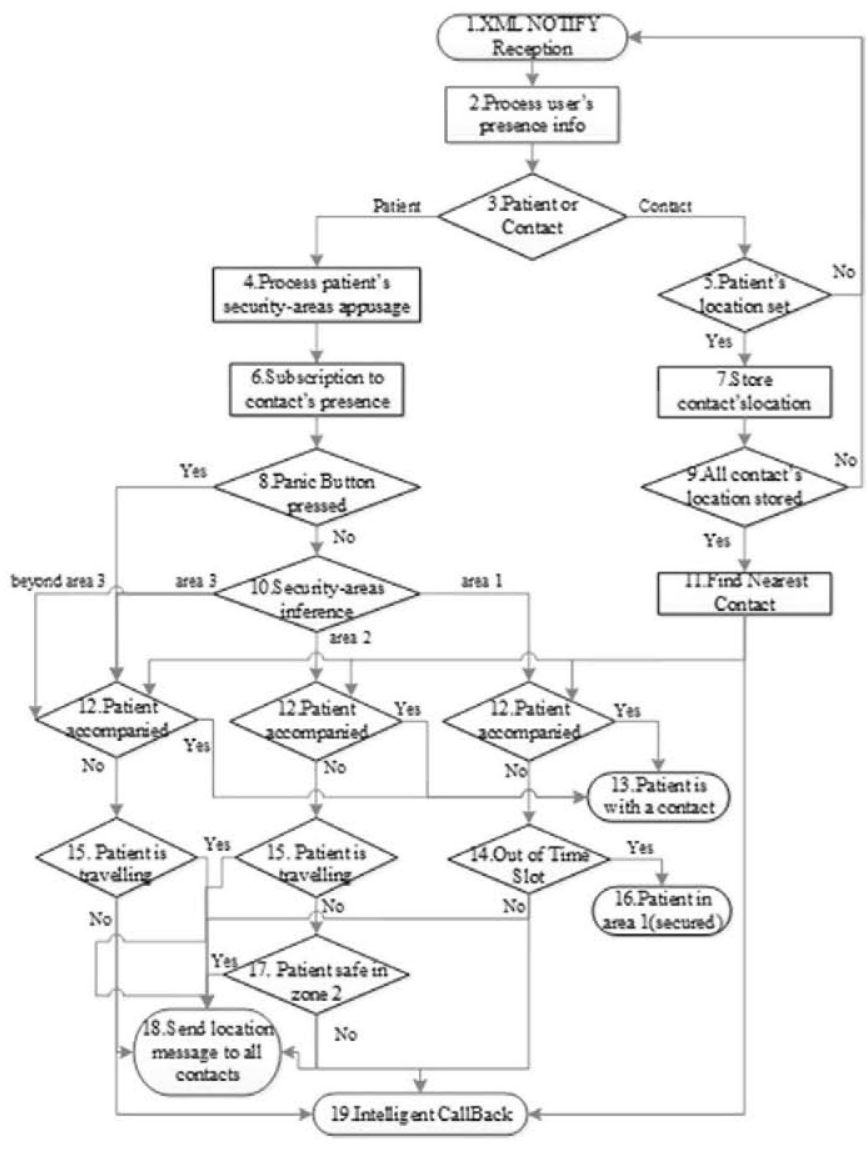

Fig. 2. Location enabler flowchart.

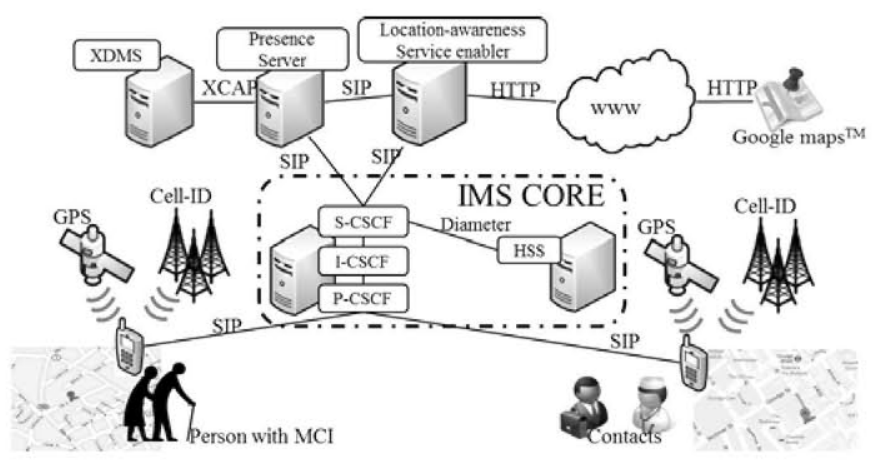

Fig. 3. Enhanced location-awareness service enabler architecture.

c) Find the Nearest Contact: After receiving the XML NOTIFY from a contact (steps 1, 2, and 3), the enabler store the contact's geolocation to calculate who will be first warned in a wandering episode (steps 5 and 7). Moreover, the enabler determines the distance between every contact registered and the patient, creating a sorted contact list (the nearest, the first) (steps 9 and 11). Thus, if an alarm is triggered, the actions executed by the enabler will involve the nearest contact as it is likely that he/she could be the first to find the patient.

d) Patient is Accompanied: The enabler can estimates if the patient is accompanied as the distance between the patient and the nearest contact has been calculated, In case of compan-
TABLE I

COMPARISON BETWEEN LBS FOR PWMCI WANDERING MANAGEMENT

\begin{tabular}{|c|c|c|c|c|c|c|c|c|c|c|}
\hline Systems & $\begin{array}{l}\frac{\infty}{0} \\
\stackrel{0}{0} \\
\stackrel{0}{0} \\
\end{array}$ & 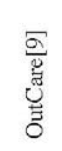 & $\begin{array}{l}\bar{\Xi} \\
\stackrel{0}{0} \\
\stackrel{0}{0} \\
\cong\end{array}$ & 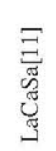 & 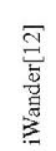 & 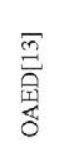 & 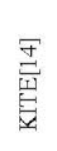 & 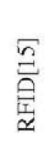 & $\frac{\bar{b}}{\frac{1}{2}}$ & 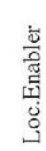 \\
\hline
\end{tabular}

SIP presence standards use -

Contacts' presence

Nearest contact

Panic button

Security areas

Time slots

Confirm location

Location message

Intelligent callback

Travelling function

Location

Route prediction

GPS accuracy mgmt.

Itinerary tracking

3-way call

" $\sqrt{ }$ " = supported, "-" = not supported, "O" = outdoor, "I" = indoor, and "?" = future support.

ion, the actions to take in any of the security areas will not be considered (steps 12 and 13).

e) Panic Button Function: The enabler offers to PwMCI who are in a safe zone (area 1) and feel an emergency situation the possibility to activate a direct and fast communication with their contacts. Automatically, the enabler will proceed as the patient would be in the area 3 (step 8 ).

f) Security Areas Inference: Once the security-areas structure have been extracted from the appusage (step 4), the enabler determines the area where the patient is. If the distance between the patient's and the hot-spot's location is under $<$ radius $>$ value, between $<$ radius $>$ and $<$ radiusTwo $>$, or among $<$ radiusTwo $>$ and $<$ radiusThree $>$, the patient will be in area 1,2 or 3, respectively. If the distance exceeds $<$ radius Three $>$, the enabler will act as if the patient is in area 3 because the risky situation is maintained (step 10).

g) Time Surveillance: Although the area 1 does not imply any hazardous situation, the PwMCI could be moving in not recommendable hours (e.g., night time). By using the presence information label <timestamp>, the enabler detects if the patient is out of the period established by the field "timeslot" of the appusage. In case the patient will be out of this period, the enabler will notify patient's contacts (steps 14,16, 18, and 19).

h) Traveling Detection: The speed of $5556 \mathrm{~m} / \mathrm{s}(20 \mathrm{~km} / \mathrm{h}$ or $12.4 \mathrm{~m} / \mathrm{h}$ ) has been set as a threshold to detect if the patient is traveling by checking the label <dyn:speed $>$ in presence information. Although the patient is in area 2 or 3 in this case, the contacts will only receive a message (step 15).

i) Confirm Location in Area 2: The patient could be in area 2 without involving a potential wandering episode. Thus, the patient is asked if he/she feels safe in this location or if he/she needs help. In case the patient feels safe, the patient's contact will only receive the patient's location. Otherwise, the 
enabler will establish a call between contacts and patient (steps 17, 18, and 19).

j) Sending Location Messaging: When an incident is detected (e.g., patient is out in night time, he needs help, or he is in area 3), the patient's current position is sent to contacts through a message that includes a link to Google Maps web page (step 18).

k) Intelligent Callback: As final stage of the wandering alarm, a SIP call between the PwMCI and the nearest contact is established. Since contacts are organized in an array by the nearest distance criteria, if the nearest contact does not answer the next contact will be called. To avoid patient's waiting, he/she is not called until the contact's answer (19). The call could support audio and video depending on the capabilities of the IMS client employed by the users (patient and contacts) as well as the user's preference. As the video is a reasonable approach to maintain older adult's peace of mind and satisfaction [23], the video call would allow a PwMCI in a wandering episode to see their contact in a display during the call and get relaxed until they are found.

\section{LBS Comparison Procedures}

A literature search has been accomplished to review solutions aimed at managing wandering episodes of PwMCI developed between 2005 and 2013. The search was held by one person using "Google scholar" and "ISI Web of Knowledge" databases. Search terms were: "wandering," "spatial disorientation," dementia," "elderly," "context awareness," and "MCI". Therefore, nine relevant solutions that have been described in Section II were identified [8]-[16]. Their main functionalities were extracted and compared with those offered by the service enabler presented.

\section{E. Implementation and Performance Test Methodology}

A set of experiments has been conducted for testing the feasibility, usability, and robustness of the enabler in a real scenario. To implement the components of IMS Core, the open-source tool OpenIMS [24] was employed. Meanwhile, the open source suite Mobicents [25] includes a XDMS server, which allows developing the security-area appusage and providing a presence information management support. Besides, Mobicents includes a SIP Servlet which was used to deploy the location-awareness enabler in the IMS architecture.

On the device side, a group of three Android smartphones with GPS support were used: Samsung Galaxy S2, Sony Xperia Arcs, and HTC Desire. Respectively, the versions of Android installed were: 4.0, 2.3, and 2.2. GPS location technique was selected because of its extended use and its position measurement robustness as any further computation is required. However, the location information could also be extracted using the Cell-ID mechanism provided by Android but the outdoor location accuracy would decrease. Specifically, we used the IMS Droid [26] client for Android, which was enhanced by developing GPS location support and IMS presence features. Moreover, this client allows the establishment of either a video call or an audio call. In both cases (PwMCI and contacts), the election of call type is simple for the users because he/she could choose the type of media by pressing a button only once when accepting the call.

Finally, the enabler's performance was assessed in every security area with the different steps showed in the flowchart (panic button pressed, patient companion, patient traveling, patient out of security time slot, message and callback sending, etc.). In addition, three mobile phones were registered, one as a patient and two as patient's contacts. The success rates of every particular enabler's function, as well as the positive false and negative false rates were evaluated.

\section{RESULTS}

\section{A. Architecture of Location-Awareness Service Enabler}

The structure of the location-awareness enabler, shown in Fig. 3, follows the LBS components requirements: locationbased applications on mobile devices, location technology, location management platforms, and server-side daemons.

The users (both person with MCI and contacts) must employ a smartphone with GPS and Cell-ID location capabilities, and a nonspecific IMS client with presence support to insert the user's location extracted in the presence information. Since the location enabler is compliant with PDIF and GEOPRIV standards, which are independent on location technology, outdoor and indoor performance is achieved.

The IMS core is responsible of managing the SIP signaling between users' devices, the presence server (PS) and the location enabler. Moreover, the enabler triggers the filters to receive the presence information. The PS handles the SIP/SIMPLE messages updating the user's presence information. By using XCAP protocol with the XDMS server, the PS updates the user profile information considering an appusage previously stored. Additionally, the enabler receives user's presence information and detects a wandering situation by examining the patient's profile. The enabler service uses the Google Maps api to show the patient's location.

\section{B. Comparison between LBS for Wandering Support}

The enabler's dynamic alerts are based on multiple factors of user's context (contacts companion, travelling, safety sense, time, etc.), and indicate a noticeable advance respect to the reviewed related work, specially the nearest contact. Moreover, the personalized security areas, the intelligent management of contact to alarm, and the inconspicuous location monitoring imply a contribution to current LBS, as well as an advance to patient's independence and autonomy support. Thus, a comparison between reviewed solutions' functionalities and the enabler's ones is shown in Table I. Furthermore, the enabler handles the standards (SIP, PIDF, and GEOPRIV) that would enhance the interoperability with other services.

\section{Implementation and Performance}

As previously mentioned, the enabler's performance was assessed in every security area combined with the different steps showed in the flowchart. Each use case was proved five times 
TABLE II

PERFORMANCE OF ENABLER's FUNCTIONS

\begin{tabular}{lccc}
\hline \multicolumn{1}{c}{ Enabler's functions } & \% success & \%False positive & \% false negative \\
\hline 1.Automatic patient's subscription & 96,11 & - & 3,89 \\
2. Users' information processing & 86,92 & - & 12,25 \\
3.Find the Nearest Contact & 75,97 & - & 23,20 \\
4. Patient is accompanied & 52,70 & 3,05 & 44,19 \\
5. Panic Button Function & 100 & - & - \\
6. Security areas inference & 94,05 & - & 5,06 \\
7. Time Surveillance & 92,71 & - & 7,29 \\
8. Travelling detection & 70,98 & 11,67 & 19,02 \\
9. Confirm location in area 2 & 75,00 & - & 25 \\
10. Sending location messaging & 75,21 & - & 24,79 \\
11. Intelligent Callback & 80,00 & - & 20 \\
\hline \hline
\end{tabular}

TABLE III

ACTIONS TO ACTIVATE ENABLER's FUNCTIONALITIES

\begin{tabular}{cl}
\hline \hline Click actions & Enabler's Functionalities Identifiers (see Section IV B) \\
\hline 0 & $\mathrm{a}, \mathrm{b}, \mathrm{c}, \mathrm{d}, \mathrm{f}, \mathrm{g}, \mathrm{h}$ \\
1 & $\mathrm{k}$ (Patient answers the call); i (Patient answers is safe) \\
2 & e(Patient activates panic button and answers the call), \\
& (Patient answer is not safe and answer the call) \\
3 & $\mathrm{j} \& \mathrm{k}$ (Contact opens message with patient's location, \\
& accesses to Google Maps link, and answers the call) \\
\hline \hline
\end{tabular}

at least reaching up to 81 tests that were carried out in $21 \mathrm{~h}$ approximately. The results are shown in Table II.

With the exception of patient companion and patient traveling detection, every function has shown a success rate higher than $75 \%$. As the GPS device sensor's accuracy was $30 \mathrm{~m}$ and it exceeds the companion threshold established $(5 \mathrm{~m})$, the patient companion failed in a $47 \%$ of the total cases. Thus, the false negative error rate reached up to $44 \%$ of the cases producing false alarms when the patient was accompanied. Furthermore, the GPS sensor accuracy affected the patient traveling function, which worked in $70 \%$ of total cases. Besides, the $20 \%$ of cases of intelligent callback did not succeeded when it was needed due to problems experienced with GPS signal acquisition of the third device (HTC Desire). Thus, the location information was not included in the presence document. Consequently, the enabler could not process user's location and transference call to second closest contact. It is expected by using other mobile device with better performance the error would be reduced.

Regarding usability and technical issues, both patient and contacts activated certain location-enabler's functions by clicking between 0 and 3 times as Table III shows. Moreover, 16 tests were run combining devices with different connections (WIFI or $3 \mathrm{G}$ ) and no difference was found in the enabler performance. In addition, the IMS architecture rejects a corrupt XML documents and informs the client about the error by means of a SIP message.

Finally, certain PwMCI's requirements about LBS described in Section II (no need for training, usability issues, low cost, size of equipment, and avoiding the stigma of being tagged) have been covered considering the use of current smartphones and the results of the enabler's performance.

\section{Discussions And Conclusion}

Currently, the smartphones are supplying a new infrastructure of mobiles devices and Internet services delivery. In a close future, these devices will become naturally attached to all people throughout their lives. Thanks to GPS capability of smartphones, the LBS are poised to become in the next stage of mobility innovation, and promote the user's location as a significant value for context-aware applications.

The location-awareness service enabler presented in this study tries to face the common limitations of current LBS as interoperability, usability or position issues. Consequently, a comparison table has been introduced in the results section showing the advantages of the location-awareness service enabler respect to the reviewed LBS in related works. Thanks to the IMS architecture, challenges related to network interoperability can be solved due to the benefits of seamless roaming that IMS provides. Regarding the application interoperability, the use of GEOPRIV offers a single interface guaranteeing that any application, which follows the standard, could use the enabler or integrate its functions. Thus, our server-side solution can be reused by third-party applications allowing client developers to be able to focus their effort in the application interface improving usability issues. Moreover, the enabler's location accuracy exclusively depends on the device's location technique (GPS or Cell-ID) since the enabler only need the location information under the GEOPRIV standard to perform its functions. As the experiments carried out has shown, the location-awareness enabler offers an acceptable success rate in most of its functions. However, one of the limitations of the study was the wrong performance of the specific GPS sensor integrated in the HTC Desire device, which did not allow obtaining a correct validation of intelligent callback function. Besides, the accuracy of the device's GPS sensor affected strongly the enabler reliability in patient accompaniment and traveling detection functions. Nevertheless, the accuracy of GPS sensors integrated in smartphones is expected to be increased integrating technologies as A-GPS, GLONASS or GALILEO. Thus, the performance of these particular functions will be improved diminishing the false negative rate.

By describing a new appusage related to security areas of users registered in the architecture, a contribution to "contextaware" services deployed in IMS architecture has been achieved. Thus, the service enabler adapts its alarm functions depending on the user profile and the contextual information (i.e., geolocation) included in the user's presence document. Besides, the new appusage allows defining as much as security areas needed for each usual place, where a PwMCI develop their daily activities.

The employment of IMS could improve the confidence of patients and contacts on using the service and providing their location information since the architecture is controlled by networks operators and older people normally are reluctant to adopt new entities in their use of technology. Thus, by managing efficiently subscriber location data, the enabler places network operators in the value-added role of service providers. To promote the use of the enabler, the network operators could provide users with a free IMS client, which could connect automatically to the IMS architecture. Furthermore, the patients will not even 
feel stigmatized by wearing a special aid as the location enabler developed can be used through smartphones.

As future works, the enabler is foreseen to show contacts the entire itinerary followed by a patient during a specified period of time ( $24 \mathrm{~h}$, a week, etc.). Besides, including a special media server in the architecture, the enabler will be able to establish video call between three or more users in case needed. Finally, the developed service enabler could be used in other scenarios since "hot-spots" definition allows providing users with different services considering their geographical proximity to a particular location.

\section{REFERENCES}

[1] (2013, Oct.). Ambient assisted living web page [Online]. Available: http://www.aal-europe.eu

[2] J. M. Wigg, "Liberating the wanderers: Using technology to unlock doors for those living with dementia," Sociol. Health Illness, vol. 32, no. 2, pp. 288-303, Feb. 2010.

[3] M. A. Rowe, S. S. Vandeveer, C. A. Greenblum, C. N. List, R. M. Fernandez, N. E. Mixson, and H. C. Ahn, "Persons with dementia missing in the community: Is it wandering or something unique?" BMC Geriatrics, vol. 11, no. 1, p. $28,2011$.

[4] R. Landau, G. K. Auslander, S. Werner, N. Shoval, and J. Heinik, "Families' and professional caregivers' views of using advanced technology to track people with dementia," Qualitative Health Res., vol. 20, no. 3, pp. 409-419, Feb. 2010.

[5] B. Richard, J. Winterbottom, and Y. M. Dawson, "Internet geolocation and location-based services," IEEE Commun. Mag., vol. 49, no. 4, pp. 102-108, Apr. 2011.

[6] B. Sadoun and O. A1-Bayari, "Location based services using geographical information systems," Comput. Commun., vol. 30, no. 16, pp. 3154-3160, Nov. 2007.

[7] M. N. K. Boulos,, "Location-based health information services: A new paradigm in personalised information delivery," Int. J. Health Geograph., vol. 2, no. 1, pp. 2, Jan. 2003.

[8] F. Miskelly, "Electronic tracking of patients with dementia and wandering using mobile phone technology," Age Ageing, vol. 34, no. 5, pp. 497-499, May 2005.

[9] J. Wan, C. Byrne, G. M. O'Hare, and M. J. O'Grady, "Orange alerts: Lessons from an outdoor case study," in Proc. 5th Int. Conf. Pervasive Comput. Technol. Healthcare, 2011, pp. 446-451.

[10] S. Hossain, K. Hallenborg, and Y. Demazeau, "iRoute: Cognitive Support for independent living using BDI agent deliberation," in Trends in Practical Applications of Agents and Multiagent Systems, eds., Berlin, Germany: Springer, 2011, pp. 41-50.

[11] J. Hoey, X. Yang, E. Quintana, and J. Favela, "LaCasa: Location and context-aware safety assistant," in Proc. 6th Int. Conf. Pervasive Comput. Technol. Healthcare, 2012, pp. 171-174.

[12] F. Sposaro, J. Danielson, and G. Tyson, "iWander: An android application for dementia patients," in Proc. Annu. Int. Conf. IEEE Eng. Med. Biol. Soc., 2010, pp. 3875-3878.

[13] Q. Lin, D. Zhang, X. Huang, H. Ni, and X. Zhou, "Detecting wandering behavior based on GPS traces for elders with dementia," in Proc. 12th Int. Conf. Control Autom. Robot. Vis., 2012, pp. 672-677.

[14] L. Robinson, K. Brittain, S. Lindsay, D. Jackson, and P. Olivier, "Keeping in touch everyday (KITE) project: Developing assistive technologies with people with dementia and their carers to promote independence," Int. Psychogeriatrics, vol. 21, no. 3, pp. 494-502, Feb. 2009.

[15] J. Vargas, "RFID application to control unauthorized egress of people with spatial disorientation," in Proc. IEEE 28th Annu. Int. Conf. IEEE Eng. Med. Biol. Soc., 2006, pp. 6392-6395.

[16] J. van Hoof, H. S. M. Kort, P. G. S. Rutten, and M. S. H. Duijnstee, "Ageing-in-place with the use of ambient intelligence technology: Perspectives of older users," Int. J. Med. Informat., vol. 80, no. 5, pp. 310-331, May 2011.
[17] Ø. Dale, "Usability and usefulness of GPS based localization technology used in dementia care," in Computers Helping People With Special Needs, eds. Berlin, Germany: Springer, 2010, pp. 300-307.

[18] M. E. Barachi, A. Kadiwal, R. Glitho, F. Khendek, and R. Dssouli, "An architecture for the provision of context-aware emergency services in the IP multimedia subsystem," in Proc. IEEE Veh. Technol. Conf., 2008, pp. 2784-2788.

[19] C. Baladrón, J. M. Aguiar, B. Carro, L. Calavia, A. Cadenas, and A. Sánchez-Esguevillas, "Framework for intelligent service adaptation to user's context in next generation networks," IEEE Commun. Mag., vol. 50, no. 3, pp. 18-25, Mar. 2012.

[20] G. Camarillo and M.-A. Garcia-Martin, The 3 G IP Multimedia Subsystem (IMS): Merging the Internet and the Cellular Worlds. New York, NY, USA: Wiley, 2011

[21] H. Sugano, S. Fujimoto, G. Klyne, A. Bateman, W. Carr, and J. Peterson "Presence information data format (PDIF)," IETF RFC 3863, 2004.

[22] R. Barnes, J. Winterbottom, and M. Dawson, "Internet geolocation and location-based services," IEEE Commun. Mag., vol. 49, no. 4, pp. 102 108, Apr. 2011.

[23] S. H. Kim, W. U. Koh, J. H. Rhim, M. H. Karm, H. S. Yu, B. Y. Lee, J. W. Shin, and J. G. Leem, "Preconsent video-assisted instruction improves the comprehension and satisfaction in elderly patient visiting pain clinic," Korean J. Pain, vol. 25, no. 4, pp. 254-257, 2012.

[24] (2013, Oct.). OpenIMSCore web page [Online]. Avaialble http://www.openimscore.org/

[25] (2013, Oct.). Mobicents presence server web page [Online]. Available http://www.mobicents.org/products.html last view

[26] (2013, Oct.). IMS Droid web page [Online]. Available: https://code.google.com/p/imsdroid/ last view

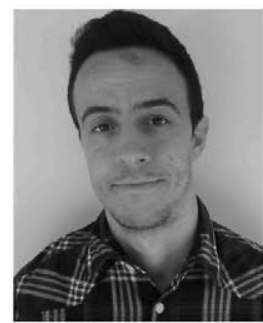

Pedro A. Moreno received the degree in telecommunication engineering in 2007, the Master's degree in biomedical engineering in 2008 , and the Ph.D. degree in 2014 from Universidad Politécnica de Madrid (UPM), Madrid, Spain.

He is currently a Researcher at the UPM. His main expertise is in the field of ambient-assisted living services for older adults and their caregivers. His current research focuses on the employment of next-generation networks technologies for supporting older adults' daily living.

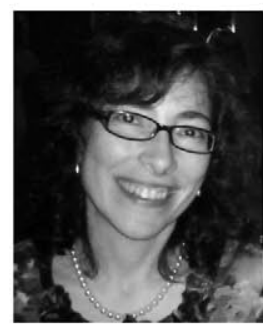

M. Elena Hernando (M'02-SM'12) received the Telecommunication Engineering and Ph.D. degrees in 1990 and 1997, respectively, from the Universidad Politécnica de Madrid (UPM), Madrid, Spain.

She is currently a Professor of biomedical engineering at the UPM. Her main expertise is in the field of telemedicine services for chronic patients and older adults. Her current research focuses on the development of services for promoting healthy lifestyle and independence living of elderly.

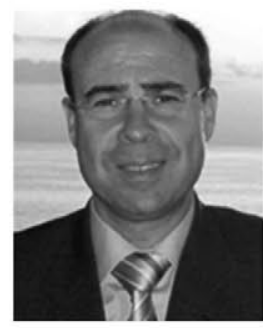

Enrique J. Gómez (S'83-M'85-SM'06) received the Telecommunication Eng. and Ph.D. degrees from the Universidad Politécnica de Madrid, Spain, in 1985 and 1987, respectively. He is a Full Professor of biomedical engineering at Universidad Politécnica de Madrid (UPM), Madrid, Spain, the Head of TF and Bioengineering Department, and the Director of MSc in Biomedical Engineering at UPM. He has published 410 publications on scientific journals and conference proceedings in related fields and directed 50 national and international research projects. His research interests include the areas of active and healthy ageing, and rehabilitation of patients with cognitive deficits. 